\title{
A saúde da população e o enfrentamento da pandemia de Covid-19 no Brasil: o esforço dos gestores e profissionais da saúde
}

\author{
Tiago de Oliveira Furlam* \\ Luciana Helena Dolabela de Abreu ${ }^{* \star}$ \\ Carla Jorge Machado ${ }^{\star \star *}$ \\ Claudia Cristina de Aguiar Pereira ${ }^{\star \star \star \star}$
}

\begin{abstract}
BRASIL. Ministério da Saúde. Conselho Nacional de Secretários de Saúde (Conass). Conselho Nacional de Secretarias Municipais de Saúde (Conasems). Covid-19: Guia orientador para o enfrentamento da pandemia na Rede de Atenção à Saúde. 4. ed. Brasília, março de 2021.
\end{abstract}

Os coronavírus representam um grupo muito diverso, entre os quais está o coronavírus da síndrome respiratória aguda grave 2 - Sars-CoV-2 - agente etiológico de uma nova doença respiratória aguda, denominada Covid-19, que emergiu no final de 2019 em Wuhan, na China (HU; ZHOU; SHI, 2020). Esse vírus apresenta alta transmissibilidade, de forma que se disseminou rapidamente pelo globo e deflagrou uma pandemia que acomete diversos países, entre eles o Brasil, um dos mais afetados pela crise sanitária, registrando quase 15 milhões de casos e mais de 400 mil mortes pela nova doença até maio de 2021 (BRASIL, 2021a). Com base no panorama exposto, documentos, diretrizes, protocolos e

\footnotetext{
*Universidade Federal de Minas Gerais (UFMG), Belo Horizonte-MG, Brasil (to.furlan@outlook.com; https://orcid.org/00000001-8826-4964).

** Universidade Federal de Minas Gerais (UFMG), Belo Horizonte-MG, Brasil (lucianahabreu@hotmail.com; https://orcid. org/0000-0003-2503-0858).

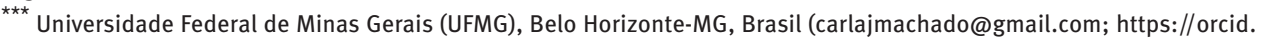
org/0000-0002-6871-0709).

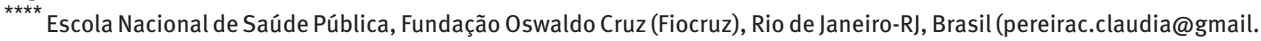
com; https://orcid.org/0000-0003-1389-9214).
} 
guias orientadores apresentam grande importância no âmbito dessa nova doença, visto servirem de referência para profissionais da saúde na luta contra uma patologia previamente desconhecida, no sentido de salvar vidas e proteger a população como um todo.

Nesse contexto, o Guia orientador para enfrentamento da pandemia na Rede de Atenção à Saúde ( $4^{\mathrm{a}}$ edição), do Ministério da Saúde, tem grande importância como material norteador das ações dos profissionais que trabalham nos diversos pontos da Rede de Atenção à Saúde (RAS). Trata-se de uma iniciativa do Ministério da Saúde (MS) em conjunto com o Conselho Nacional de Secretários de Saúde (Conass) e o Conselho Nacional de Secretarias Municipais de Saúde (Conasems). A presente edição, lançada em março de 2021, é composta por quatro capítulos, referências e nove anexos. Entre os novos assuntos incluídos nesta edição estão: novas variantes do Sars-CoV-2; descrição de casos de reinfecção; informações sobre vacinas; e atualizações quanto à avaliação de risco, ao diagnóstico e ao atendimento às gestantes.

O primeiro capítulo - “Apresentação” - traz uma breve descrição do Guia feita pelos seus elaboradores, reforçando que esse instrumento orientador foi revisado de forma a abordar as atividades que devem ser realizadas no combate à pandemia de Covid-19 nos variados pontos de atenção da RAS. Ainda são apresentados os "7 passos para APS", ou seja, os sete grandes grupos de ações básicas que devem ser norteadores do combate à pandemia na Atenção Primária à Saúde (APS), além de incluída uma descrição dos “5 passos para população", que devem ser seguidos durante o período de combate ao SarsCoV-2. Como o próprio título do capítulo indica, trata-se de uma breve apresentação ao tema, porém, com informações relevantes e descritas de maneira didática, visto que o documento versará, mais à frente, de forma mais aprofundada sobre diversos assuntos.

O segundo capítulo - "Introdução" - aborda a perspectiva social, situacional e epidemiológica do vírus Sars-CoV-2, o qual levou ao primeiro caso de Covid-19 no Brasil em 26 de fevereiro de 2020 e, com prazo de um mês, deflagrou a transmissão comunitária no país. Assim, com o decorrer da contaminação em massa desde março de 2020, descreve-se que o Brasil atualmente registra, além do expressivo número de casos e de um platô elevado de mortes diárias, uma nova variante do vírus, denominada P.1, linhagem B.1.1.28, a qual acredita-se que possui maior potencial de infectividade, sendo relacionada ao município de Manaus.

Apesar de todo esse contexto epidemiológico desfavorável, o capítulo aborda a atual campanha de vacinação nacional em curso, com vacinas aprovadas pela Agência Nacional de Vigilância Sanitária (Anvisa), como a CoronaVac e a ChAdOx1 nCoV-19, além de demais imunizantes aprovados para uso emergencial, como a vacina Janssen Covid-19. 0 capítulo mostra toda a dinâmica envolvendo a aplicação de cada tipo de vacina, bem como as precauções e recomendações existentes para cada imunizante, em cada faixa etária. Trata-se de assunto pertinente e extremamente relevante, pois a vacinação em massa é considerada um dos passos fundamentais para o controle da pandemia de Covid-19, visto ser calculado que, para obtenção de imunidade de rebanho, cerca de $70 \%$ da população 
mundial global precisaria ser vacinada (FONTANET; CAUCHEMEZ, 2020). Nesse ponto, é importante reforçar o conceito de imunidade de rebanho (RANDOLPH; BARREIRO, 2020), que é a proteção indireta contra infecção conferida a indivíduos suscetíveis quando uma proporção suficientemente grande de pessoas imunes existe em uma população. Há ainda o conceito de limiar de imunidade de rebanho, que é o ponto a partir do qual a proporção de indivíduos suscetíveis em uma população cai abaixo do limite necessário para a transmissão (RANDOLPH; BARREIRO, 2020). Assim, a vacinação em massa proporciona que se chegue mais rapidamente ao debelamento da pandemia.

Além de toda essa contextualização, o capítulo elucida o contexto da prática clínica e laboratorial, indicando e explicando os diversos testes possíveis e suas aplicações para o enquadramento dos casos de Covid-19 como suspeitos ou confirmados. Apresenta-se a abordagem técnica a ser realizada mediante sinais e sintomas dos pacientes, considerando aspectos como idade, presença de doenças crônicas e gestação. 0 Guia ainda expõe a dinâmica burocrática que envolve o sistema de notificação de casos e de óbitos, dado que essas informações são imprescindíveis para o delineamento de ações e campanhas, além de permitirem o acompanhamento da situação epidemiológica que o país se encontra, necessária para elaboração de políticas públicas assertivas e resolutivas.

O capítulo ainda apresenta os grupos considerados de risco para desenvolver as formas graves da Covid-19, entre eles os idosos, as gestantes e puérperas, as crianças e outros fatores de risco ou condições crônicas, independentemente da idade. Ademais, abordam-se também as medidas de prevenção ao contágio necessárias e que, atualmente, têm grande notoriedade, credibilidade e comprovação científica, porém baixa adesão pela população, como o uso de máscaras e o uso correto dos equipamentos de proteção individual. Outro tópico muito relevante de escolha dos autores, particularmente para gestores da saúde, refere-se às estratégias para avaliação de risco, por meio da classificação da situação epidemiológica e as consequentes medidas de distanciamento e restrições em cada caso. Além disso, discorre-se a respeito da terapia medicamentosa para o tratamento da doença, expondo as evidências existentes e os pareceres das instituições de saúde, ressaltando a legalidade do ato médico, baseado na centralização do ato do cuidar, bem como o princípio da autonomia do paciente.

Por fim, além de discorrer sobre a dinâmica no atendimento do paciente, o capítulo aborda questões referentes à existência e ao manejo do paciente com reinfecção pelo coronavírus, situação de grande importância e que deve ser notificada para controle epidemiológico. Não obstante, são esclarecidas questões relacionadas à reabilitação de pacientes que permaneceram em internações prolongadas, devido a quadro grave, expondo as intervenções necessárias para a adequada reabilitação das funções do paciente, importante para controle dos danos decorrentes da pandemia e representando a chamada prevenção terciária, cujo objetivo principal é reabilitar os doentes com patologias instaladas (OLIVEIRA; REIS, 2012). 
Assim, o capítulo explica toda a dinâmica do âmbito da saúde para o enfrentamento da pandemia do novo coronavírus. Com isso, ao evidenciar desde os dados clínicos até a dinâmica existente na RAS, o texto permite que o leitor conheça as atuais diretrizes e administração da doença existentes no país, para o manejo e a prevenção contra o Sars-CoV-2, sendo de caráter imprescindivel a trabalhadores e gestores da saúde.

0 terceiro capítulo - “As redes de atenção no enfrentamento da pandemia” - expõe a organização dos níveis de atendimento na RAS. Nesse contexto, abordagens existentes desde o domicílio, as unidades básicas de saúde até os serviços de urgência e de emergência são colocadas a partir das demandas e dos encargos presentes no âmbito da pandemia. Com isso, expõe-se a dinâmica de atendimento à saúde existente, bem como o papel de cada rede de atendimento no contexto do recebimento e do fluxo de pacientes contaminados, assunto que será mais bem abordado no capítulo seguinte. Assim, identificar papéis destinados a cada setor da RAS, a partir do cenário de descentralização preconizado pelo SUS, permite o melhor controle do cuidado em saúde, sendo esse conhecimento de suma importância para a prática e o pleno funcionamento de todos os setores de atendimento.

O quarto e último capítulo é denominado "Matrizes específicas dos diversos pontos de atenção na RAS”. Essa seção do Guia tem por objetivo tratar especificamente de cada ponto da RAS, incluindo a APS, os centros comunitários e os centros de atendimento para enfrentamento à Covid-19, a atenção ambulatorial especializada (AAE), a atenção hospitalar (AH), o Serviço de Atendimento Móvel de Urgência (Samu), as Unidades de Pronto Atendimento (UPA) e as unidades hospitalares perinatais. Trata-se, portanto, de uma abordagem essencial para o entendimento do funcionamento e das ações específicas que cada ponto da RAS é responsável, sendo útil não apenas para os profissionais da saúde de diferentes especialidades que lidam diretamente com pacientes com Covid-19, mas também para os gestores de saúde. 0 capítulo tem potencial de auxiliar no planejamento adequado que leve à clara distinção de funções na RAS, facilitando a formulação de fluxogramas de atendimento e de encaminhamentos, de forma a evitar que haja sobrecarga sobre algum serviço.

Trata-se de um capítulo muito rico em informações e com grande abrangência em seu conteúdo. Por exemplo, no que tange à APS, há descrição sobre as ações e as atividades que devem ser seguidas no atendimento às crianças, às gestantes, às puérperas, aos idosos, aos hipertensos, aos diabéticos, às pessoas em situação de rua, à população indígena, além de incluir também a atenção à saúde sexual e reprodutiva, à saúde bucal, à saúde mental e ao controle do câncer de colo uterino e de mama.

Discorre-se também sobre o papel dos centros comunitários e dos centros de atendimento para enfrentamento da Covid-19. 0 capítulo ainda contém informações sobre a $\mathrm{AAE}$, incluindo o teleatendimento, a triagem de sintomáticos, o atendimento às diversas populações, de forma semelhante àquela feita na seção sobre a APS, mas acrescentando outras condições, como o atendimento ao doente renal crônico e o atendimento oftalmológico. As ações referentes a AH, Samu e UPA foram agrupadas em um mesmo subtópico e 
incluem diversos cenários, como os fluxos de atendimentos para a $\mathrm{AH}$ na rede de urgência e de emergência, as ações nas atividades das tendas de testagem, as ações e atividades das unidades de saúde temporária para assistência hospitalar - os hospitais de campanha -, entre outros.

Finaliza-se o capítulo com informações sobre as unidades hospitalares perinatais, assunto extremamente importante para atendimento adequado e seguro a um dos grupos considerados de risco para desenvolver as formas graves da Covid-19. Entre os temas abordados, estão as ações e atividades nos hospitais, nas maternidades e nas unidades neonatais, a estrutura, a organização e os fluxos assistenciais nas unidades perinatais, as considerações pré-hospitalares na atenção hospitalar ao parto e ao nascimento, o diagnóstico da infecção no período neonatal, o plano de cuidado intra-hospitalar, o plano de alta hospitalar e a integração da atenção hospitalar à APS e a interface com a AAE, entre outros tópicos.

Logo em seguida, os autores disponibilizam as referências bibliográficas, estruturadas por capítulo, utilizadas para elaboração do documento e os nove anexos contidos no Guia.

De forma geral, os anexos versam sobre assuntos relevantes, como, por exemplo, a telemedicina e a teleassistência, que se tornaram essenciais para manutenção da assistência durante o período de isolamento social. Há disponibilização de instrumentos práticos e visuais, como checklists, fluxogramas, além de quadros e tabelas sobre os diversos assuntos, que auxiliam e facilitam no entendimento do tema por parte dos leitores. Como exemplo, pode ser citado o script para aplicação do Índice de Vulnerabilidade Clínico Funcional-20 (IVCF-20) por telefone, instrumento extremamente útil para rastreio de idosos em risco de fragilização ou já frágeis (MORAES et al., 2015).

Para além da saúde dos indivíduos e da saúde coletiva, o material apresentado no Guia pode servir de referência para modelos populacionais de controle da pandemia, tanto no Brasil quanto no mundo, pois, ao expor fatores que interferem no ritmo da pandemia e no seu desdobramento, lança luz a novas possibilidades de análise e novas abordagens das variáveis envolvidas. Esses modelos têm sido buscados em várias partes do mundo, com proposições de estratégias para o enfrentamento da pandemia (ABIDEMI et al., 2021). No Brasil, por exemplo, o papel dos centros comunitários ou do planejamento adequado dos atendimentos e encaminhamentos pode ser explorado num modelo matemático a ser aplicado em grandes populações, visando buscar o funcionamento ótimo das variáveis de todo o sistema para auxílio no controle da pandemia. Com efeito, estratégias de redução de novos casos devem ser concebidas em termos de ações individuais e coletivas, que envolvem ações coordenadas de saúde.

Finalmente, o Guia orientador para o enfrentamento da pandemia na Rede de Atenção à Saúde (4a edição), ao abordar conteúdos cientificamente comprovados e referenciados, constitui um instrumento confiável e seguro como norteador no contexto da pandemia e supre uma carência na ausência de protocolos assistenciais mais claros e unificados. Enfim, trata-se de um documento de grande relevância, completo, didático e bem estruturado, o 
qual é importante acréscimo à literatura e será extremamente útil para os mais diversos públicos, desde os profissionais e os gestores da área da saúde até os estudantes interessados na saúde das populações e, claro, de interesse para a própria população em geral.

\section{Referências}

ABIDEMI, A.; ZAINUDDIN, Z. M.; AZIZ, N. A. B. Impact of control interventions on COVID-19 population dynamics in Malaysia: a mathematical study. The European Physical Journal Plus, n. 136, article 237, 2021. Disponível em: https://link.springer.com/article/10.1140/epjp/s13360021-01205-5.

BRASIL. Ministério da Saúde. Covid-19 no Brasil. 2021a. Disponível em: https://susanalitico. saude.gov.br/extensions/covid-19_html/covid-19_html.html.

BRASIL. Ministério da Saúde. Conselho Nacional de Secretários de Saúde (Conass). Conselho Nacional de Secretarias Municipais de Saúde (Conasems). Covid 19: Guia orientador para o enfrentamento da pandemia na Rede de Atenção à Saúde. 4. ed. Brasília, 2021b. Disponível em: https://www.conasems.org.br/wp-content/uploads/2021/04/Covid-19_guia_orientador_4ed. pdf.

FONTANET, A.; CAUCHEMEZ, S. COVID-19 herd immunity: where are we? Nature Reviews Immunology, n. 20, p. 583-584, 2020. Disponivel em: https://www.ncbi.nlm.nih.gov/pmc/ articles/PMC7480627/.

HU, B.; GUO, H.; ZHOU, P.; SHI, Z. L. Characteristics of SARS-CoV-2 and COVID-19. Nature Reviews Microbiology, n. 19, p. 141-154, 2020. Disponivel em: https://www.nature.com/articles/s41579020-00459-7.

MORAES, E. N.; CARMO, J. A.; MORAES, F. L.; AZEVEDO, R. S.; MACHADO, C. J.; MONTILLA, D. E. R. Índice de Vulnerabilidade Clínico Funcional-20 (IVCF-20): reconhecimento rápido do idoso frágil. Revista de Saúde Pública, n. 50, 2016. Disponível em: http://www.scielo.br/scielo. php?script=sci_arttext\&pid=S0034-89102016000100254\&lng=pt\&nrm=iso..

OLIVEIRA, C. C.; REIS, A. Questões epistemológicas e bioéticas da prevenção quaternária. Physis, n. 22, p. 1485-1502, 2012. Disponivel em: https://doi.org/10.1590/S0103-73312012000400012.

RANDOLPH, H. E.; BARREIRO, L. B. Herd immunity: understanding COVID-19. Immunity, n. 52, p. 737-741, 2020. Disponivel em: https://pubmed.ncbi.nlm.nih.gov/32433946/.

\section{Sobre os autores}

Tiago de Oliveira Furlam é acadêmico de graduação do curso de Medicina da Faculdade de Medicina da Universidade Federal de Minas Gerais (UFMG).

Luciana Helena Dolabela de Abreu é acadêmica de graduação do curso de Medicina da Faculdade de Medicina da Universidade Federal de Minas Gerais (UFMG).

Carla Jorge Machado é Ph.D pela Johns Hopkins University. Professora titular do Departamento de Medicina Preventiva e Social da Universidade Federal de Minas Gerais (UFMG).

Claudia Cristina de Aguiar Pereira é doutora em Population Health pela University of Wisconsin, Madison. Pesquisadora do Departamento de Administração e Planejamento em Saúde da Escola Nacional de Saúde Pública Sergio Arouca (Ensp) da Fundação Oswaldo Cruz (Fiocruz). 


\title{
Endereço para correspondência
}

\author{
Tiago de Oliveira Furlam \\ Av. Prof. Alfredo Balena, 190, Centro \\ 30130-100 - Belo Horizonte-MG, Brasil \\ Luciana Helena Dolabela de Abreu \\ Av. Prof. Alfredo Balena, 190, Centro \\ 30130-100 - Belo Horizonte-MG, Brasil \\ Carla Jorge Machado \\ Av. Prof. Alfredo Balena, 190, Centro \\ 30130-100 - Belo Horizonte-MG, Brasil \\ Claudia Cristina de Aguiar Pereira \\ Rua Leopoldo Bulhões, 1480, $7^{\circ}$ andar, Manguinhos \\ 21041-210 - Rio de Janeiro-RJ, Brasil
}

Recebido para publicação em 02/05/2021

Aceito para publicação em 02/06/2021 


\section{ERRATA}

No título, página 1, onde se lia:

"A saúde da população e o enfretamento da pandemia de Covid-19 no Brasil: o esforço dos gestores e profissionais da saúde"

Leia-se:

"A saúde da população e o enfrentamento da pandemia de Covid-19 no Brasil: o esforço dos gestores e profissionais da saúde"

No cabeçalho, nas páginas 2, 3, 4, 5, 6 e 7, onde se lia:

"A saúde da população e o enfretamento da pandemia de Covid-19 no Brasil

Leia-se:

"A saúde da população e o enfrentamento da pandemia de Covid-19 no Brasil"

Revista Brasileira de Estudos de População (2021), v. 38, e0158 